hep-th/9512196

TIFR/TH/95-64

\title{
Orbifolds of M-theory
}

\author{
Keshav Dasguptal and Sunil Mukhil \\ Tata Institute of Fundamental Research, \\ Homi Bhabha Rd, Bombay 400 005, India
}

\begin{abstract}
We study $Z_{2}$-orbifolds of 11-dimensional M-theory on tori of various dimensions. The most interesting model (besides the known $S^{1} / Z_{2}$ case) corresponds to $T^{5} / Z_{2}$, for which we argue that the resulting six-dimensional theory is equivalent to the type IIB string compactified on K3. Gravitational anomaly cancellation plays a crucial role in determining what states appear in the twisted sector. Most of the other models appear to break spacetime supersymmetry. We observe that M-theory tends to produce chiral compactifications on orbifolds, and that our results may provide an insight into the mechanism by which twisted-sector states arise in this hypothetical theory.
\end{abstract}

December 1995

1 E-mail: keshav@theory.tifr.res.in

2 E-mail: mukhi@theory.tifr.res.in 


\section{Introduction}

An 11-dimensional supersymmetric theory of gravity appears to exist from which many (perhaps all) properties of string theory can be extracted[1-19]. This theory (currently called "M-theory") has 11-dimensional supergravity as its low energy effective field theory, and does not appear to be described by strings. Certain properties of M-theory can be described in terms of extended objects, the twobranes and fivebranes, much in the same way that properties of various string theories can be described by $p$-branes for various $p$.

Because M-theory is not a string theory, most of its compactifications that have been studied are geometric in nature, since the analogue of conformal field theory (if any) relevant to this case is not known. These include compactifications on tori, K3 [20] [4] [ [ CalabiYau spaces [21] 22] 223], and other manifolds of exceptional holonomy [24]. One notable exception appeared in recent work of Horava and Witten[14], where a proposal was given for the compactification of M-theory to 10 dimensions on the orbifold $S^{1} / Z_{2}$. This construction, while closely following an earlier construction of "orientifolds" [25] [26] in closed string theories, is necessarily more difficult to study since the properties of M-theory responsible for non-geometrical effects are presently unknown. Nevertheless, a number of consistency conditions enabled the authors of Ref. [14 to conclude that there should be a sensible orientifold of M-theory on $S^{1} / Z_{2}$, leading to the $E_{8} \times E_{8}$ heterotic string in 10 dimensions.

In the following, we will explore other ways of orbifolding and orientifolding M-theory, with a view to learning what kind of non-geometric behaviour of the underlying theory can be inferred. Consistency conditions will as usual be crucial in drawing conclusions for which calculational methods are as yet unavailable; these conclusions will of course be worth testing should such methods become available in the future.

It is appropriate to briefly review the orbifold/orientifold ideas [25] [26] which played an important role in Ref. [14]. The basic observation is that an orientation-reversing $Z_{2}$ transformation of oriented closed string world sheets is a symmetry in 10-dimensional type IIB string theory, and can be realized also on type IIA string theory if additionally one spacetime dimension is compactified and changes sign under the $Z_{2}$ action. In the former case one discovers a theory of unoriented closed strings (from the untwisted sector, by projecting onto the $Z_{2}$-invariant states) along with unoriented open strings carrying $S O(32)$ Chan-Paton factors (from the twisted sector). In the latter case the situation is more subtle: first of all one should compactify to 9 dimensions on a circle, then take the $Z_{2}$ 
quotient of the circle simultaneously with orientation reversal of the world sheet. The result is again a theory of unoriented closed strings in the untwisted sector, but the twisted sector states are open strings with Dirichlet boundary conditions which constrain their ends to lie on 8-branes in space, transverse to the two fixed points of the 10th direction. These open strings carry $S O(16)$ Chan-Paton factors and produce a gauge group $S O(16) \times S O(16)$, one factor from each fixed point.

The two orientifold theories described above become equivalent when viewed in 9 dimensions, after turning on a Wilson line in the former. The moduli spaces are identical although Wilson lines in the former theory correspond to shifting the end points of the open strings in the latter.

The above picture emerges after explicit calculation of amplitudes which have a simultaneous interpretation as closed-string tree diagrams and open-string one-loop diagrams. Comparing the two calculations allows one to deduce the presence of a degeneracy which is then interpreted in terms of Chan-Paton factors. Since analogous calculations are inaccessible in M-theory, one may wonder how to study the twisted sector in an orientifold of it (not to mention that one doesn't know how to define the analogue of orientation-reversal, to start with).

The key to obtaining a consistent picture comes from two important facts. The field content of massless modes of M-theory is in correspondence with that of type IIA string theory in 10 dimensions, so that one can define an orientifold by imitating its known action on the spacetime fields and spacetime coordinates of the IIA theory. Second, the nature of the twisted sectors can be inferred by requiring the cancellation of gravitational anomalies in 10 dimensions and taking into account the multiplet structure of the residual supersymmetry. Thus one starts with 11-dimensional supergravity, having spacetime bosonic fields $g_{M N}$ and $A_{M N P}$, compactifies the 11 th direction $X^{11}$ on a circle of radius $R_{11}$, and considers the $Z_{2}$ action

$$
\begin{gathered}
A \rightarrow-A \\
X^{11} \rightarrow-X^{11}
\end{gathered}
$$

The $Z_{2}$ invariant modes in 10 dimensions are $g_{\mu \nu}, g_{11,11}$ and $A_{\mu \nu, 11}$, or in other words a metric, scalar and 2-form in 10d. This is precisely the bosonic content of the (chiral) $N=1$ supergravity multiplet in 10d. Since this multiplet suffers from a gravitational anomaly, one expects twisted-sector states to arise precisely in the right combination to cancel it. Such states must lie in chiral $N=1$ gauge multiplets, and there must be two copies (one 
at each fixed point), which uniquely determines the gauge group to be $E_{8} \times E_{8}$. Many checks of this picture were made in Ref. [14] and they all tend to confirm it.

This does not tell us how the gauge groups actually arise in M-theory, though we will speculate on that later. In the following, we first investigate other situations where something can be said about orbifolds of M-theory.

\section{2. $T^{5} / Z_{2}$ Orbifold of M-theory, and a puzzle}

Besides the example above, gravitational anomalies can only help us if we compactify M-theory to 6 or 2 spacetime dimensions. Accordingly we first investigate the orientifold down to 6 dimensions on $T^{5} / Z_{2}$. As for the $S^{1} / Z_{2}$ case, the 3 -form changes sign under the reversal of the five coordinates $X^{7}, X^{8}, X^{9}, X^{10}, X^{11}$. The result is the following set of massless $Z_{2}$-invariant fields:

$$
\begin{aligned}
g_{M N} & \rightarrow g_{\mu \nu}, g_{i j}=15 \phi \\
A_{M N P} & \rightarrow A_{\mu \nu i}=5 B_{\mu \nu}, A_{i j k}=10 \phi
\end{aligned}
$$

Thus the total bosonic spectrum in the untwisted sector is a metric, 52 -forms and 25 scalars. Remarkably, this is enough to tell us that we are dealing with the chiral $N=4$ supersymmetry in 6 dimensions, since that is the only one for which the above spectrum can be decomposed into multiplets! Of course, this is consistent with the expectation that the orientifold actually reverses the sign of half the components of the 11-dimensional gravitino, leaving four chiral symplectic Majorana-Weyl (SMW) gravitinoes and 20 SMW fermions of the opposite chirality. (Note that this supersymmetry is $N=4$ in SMW units and leads to $N=4$ supersymmetry on toroidal compactification to four dimensions. In the literature it is equally often called $N=2$, counted in units of Weyl spinors.)

Indeed, the spectrum obtained above decomposes into a chiral supergravity multiplet

$R(G)=\left(g_{\mu \nu}, B_{\mu \nu}^{(i j)(-)}\right)$ (we list only the bosons, the $(-)$ superscript indicates an anti-selfdual two-form, and $(i j)$ is a composite index representing the 5 of $U S p(4))$ and five matter multiplets $R(M)=\left(B_{\mu \nu}^{(+)}, \phi^{(i j)}\right)$. We now have a situation very analogous to that in $10 \mathrm{~d}$, since the above structure suffers from a gravitational anomaly, and there is a unique way to eliminate the anomaly. This corresponds to adding 16 more matter multiplets $R(M)$. The resulting spectrum is that of the type IIB supergravity compactified on K3[27], whose lowenergy multiplet structure is the unique one consistent with chiral $N=4$ supersymmetry and anomaly cancellation. 
We would therefore conclude that the "twisted sector" of M-theory on $T^{5} / Z_{2}$ provides precisely 16 matter multiplets $R(M)$, and that the resulting theory is equivalent to the type IIB string on K3. Before going on to test this hypothesis, however, we are faced with an immediate puzzle. The torus $T^{5} / Z_{2}$ has 32 fixed points. Taking a regular torus with all sides equal, one would expect the anomaly to be distributed equally among the fixed points (and perhaps described by open membranes with Dirichlet boundary conditions which attach their boundaries to the fixed points). But even with our limited understanding of M-theory, it seems impossible to obtain 16 multiplets equally distributed among 32 fixed points, at least without violating manifest supersymmetry and Lorentz invariance in the formalism.

To investigate this point in more detail, consider the further compactification to 5 dimensions on $S^{1}$. On one side, we have M-theory on $T^{5} / Z_{2} \times S^{1}$, while on the other we expect type IIB on $K 3 \times S^{1}$. The latter theory (for generic K3) gives a massless spectrum containing the $N=4$ supergravity multiplet in $5 \mathrm{~d}$, along with 21 copies of the $N=4$ Maxwell multiplet. For the former, we can reverse the order of compactification. Mtheory on $S^{1}$ gives the type IIA string in $10 \mathrm{~d}$, and we should now make a $T^{5} / Z_{2}$ orientifold of that to five dimensions and compare.

This time we know what to do, since orientifolds of the type IIA string are well-defined - indeed, they are precisely the ones which inspired the similar construction in $11 \mathrm{~d}$ that we are attempting to pursue. It has been argued in Ref. 25 that the orientifold of the type $I I A / B$ string on $T^{n} / Z_{2}$ (IIA for odd $n$ and IIB for even $n$ ) gives a theory, called type $\mathrm{I}^{\prime}$, whose twisted sector contains open strings with the Chan-Paton factors of $S O\left(32.2^{-n}\right)$. Since there are $2^{n}$ fixed points, the full Chan-Paton group is $\left(S O\left(32.2^{-n}\right)\right)^{2^{n}}$. The rank of this group is formally $\frac{1}{2} \cdot 32 \cdot 2^{-n} \cdot 2^{n}=16$ independent of $n$, but actually this is valid only for $n \leq 6$. For $n=5$ it appears that the Chan-Paton group has become $(S O(1))^{32}$, and $S O(1)$ strings have no massless states (they do have massive ones, at odd levels 28). So from this discussion it appears that the twisted sector of the type IIA string on $T^{5} / Z_{2}$ contributes no massless multiplets. Then in comparing the massless spectrum to type IIB on $K 3 \times S^{1}$, we would find it is missing precisely 16 Maxwell multiplets. This appears to violate the proposed equivalence of M-theory on $T^{5} / Z_{2}$ to IIB on K3.

This picture cannot be quite right, for the following reason. The orientifold on $T^{5} / Z_{2}$ of type IIA is, more or less by definition, the T-dual of the type I open string on $T^{5}$, when all the 5 directions are dualized. The latter theory can certainly have rank-16 gauge groups (Abelian or non-Abelian) in 5d, so it must be possible for the former to have them 
as well. The only thing that is impossible in $5 \mathrm{~d}$ or less is to have symmetrically distributed Chan-Paton factors over 32 or more fixed points in the type $\mathrm{I}^{\prime}$ description. Indeed, the analysis of Chan-Paton factors on orientifolds in Refs. 29] and [30] implies that type I' strings always have $U(1)^{16}$ Chan-Paton factors, which can be distributed in various ways on the orbifold, and give rise to unitary enhanced symmetries whenever they merge, and even larger orthogonal groups when they merge at fixed points.

Nevertheless, the question arises why M-theory produces only 16 matter multiplets in $6 \mathrm{~d}$, the unique number allowed by gravitational anomaly cancellation, despite the presence of 32 fixed points. We will now propose a resolution to the puzzle.

\section{Couplings and Radii}

Let us assume despite the apparent puzzle found above, that $\mathrm{M}$ theory on $T^{5} / Z_{2}$ is equivalent to the type IIB string on K3. Since M theory has no moduli or couplings, all the parameters of the resulting $6 \mathrm{~d}$ theory will come from the toroidal orbifold. On the IIB side, there is a moduli space labelled by $O(21,5, R) / O(21, R) \times O(5, R)$ 31 32]. The duality group acting on this is $O(21,5, \mathbb{Z})$, and this includes, among other things, strong-weak coupling duality transformations on the coupling constant in 6 dimensions.

We will find a relation between the M-theory radii and this coupling constant, and check it in two different ways. This will enable us to propose a resolution to the puzzle encountered in the previous section.

M-theory compactified on a circle of radius $R_{11}$ is believed to be equivalent to type IIA string theory in 10d[3] [4] with the string coupling $\lambda_{10}$ given by [4]:

$$
\lambda_{10}^{2 / 3}=R_{11}
$$

If we further compactify the IIA theory on $T^{4}$ to 6 dimensions, the coupling constant in $6 \mathrm{~d}$ is given by the usual relation

$$
\lambda_{6}=\frac{\lambda_{10}}{\sqrt{\operatorname{vol}\left(T^{4}\right)}}
$$

Assume a rectangular torus. The compactification radii $R_{i}, i=7, \ldots, 10$ as measured in

the M-theory metric are related to the radii $R_{i}^{(10)}$ measured in the string metric of the IIA theory by:

$$
R_{i}=\frac{R_{i}^{(10)}}{\lambda_{10}^{1 / 3}}
$$


as follows from the Weyl scaling between the two metrics. The volume of $T^{4}$ in Eq.(3.2) above is the one measured in the string metric, so we find

$$
\lambda_{6}=\frac{\lambda_{10}^{1 / 3}}{\left(R_{7} R_{8} R_{9} R_{10}\right)^{1 / 2}}=\left(\frac{R_{11}}{R_{7} R_{8} R_{9} R_{10}}\right)^{1 / 2}
$$

Now in this discussion we have been working with a torus - and not orbifold - compactification of M-theory. But relations between couplings and radii such as the ones derived above are insensitive to orbifolding, so we can conclude that the above relations hold also for M-theory on $T^{5} / Z_{2}$ and hence that $\lambda_{6}$ is the coupling of the type IIB theory on K3. The asymmetry in the five radii of the torus-orbifold will have a natural interpretation in terms of the type IIB theory, as we will see below.

One way to check the above relation, and our proposal for the M-theory orbifold in general, is the following. If the M-theory on $T^{5} / Z_{2}$ is indeed equivalent to type IIB on K3, then the equivalence must be present on further compactification of both sides on $S^{1}$, as mentioned before. The type IIB theory on $K 3 \times S^{1}$ has been argued $\mathbb{4}$ to be equivalent to the heterotic string toroidally compactified to 5 dimensions, with the identification

$$
R_{h, 6}=\frac{1}{\lambda_{B, 6}}
$$

where the $h$ and $B$ subscripts refer to the heterotic and type IIB strings respectively.

On the other side, we get a type I' theory of open and closed strings. Relationships between toroidally compactified type I, type I' and heterotic strings have been recently explored[30]. They are all equivalent, with the maps being T-duality from type $\mathrm{I}^{\prime}$ to type I, and strong-weak coupling duality from type I to heterotic 33] [34]. One can derive relationships between the radii and coupling constants of type $\mathrm{I}^{\prime}$ and heterotic strings using type I as an intermediate step. For $n$ compactified dimensions, the result is (see Eq.(21) of Ref. 30)

$$
\left(R_{I^{\prime}, i}\right)^{n-2}=\lambda_{I^{\prime}, 10} \frac{\prod_{i=11-n}^{10} R_{h, i}}{\left(R_{h, i}\right)^{n-2}}
$$

This is easily inverted to express each heterotic string radius in terms of all the type $\mathrm{I}^{\prime}$ radii and the $10 \mathrm{~d}$ coupling:

$$
R_{h, i}=\left(\frac{\prod_{i=11-n}^{10} R_{I^{\prime}, i}}{\lambda_{I^{\prime}, 10}}\right)^{1 / 2} \frac{1}{R_{I^{\prime}, i}}
$$


As before, the radii $R_{I^{\prime}, i}$ are related to those measured in the M-theory by $R_{I^{\prime}, i}=\lambda_{I^{\prime}, 10}^{1 / 3} R_{i}$. Inserting this, we find in particular that

$$
R_{h, 6}=\left(\frac{\prod_{i=11-n}^{10} R_{i}}{R_{6}}\right)^{1 / 2} \lambda_{I^{\prime}, 10}^{(n-5) / 6}
$$

For $n=5$, the case at hand, the coupling constant dependence neatly drops out. Finally, we recall that what we are calling $R_{6}$ here is one of the radii of the 5 -torus orbifold, which has been exchanged with $R_{11}$ when we interchanged the orders of compactification on $T^{5} / Z_{2}$ and $S^{1}$. Thus we should now rename $R_{6}$ as $R_{11}$. Combining Eqs.(3.8) and (3.5) then leads to

$$
\lambda_{B, 6}=\left(\frac{R_{11}}{R_{7} R_{8} R_{9} R_{10}}\right)^{1 / 2}
$$

in perfect agreement with Eq.(3.4) above.

The asymmetry in the above relation, alluded to earlier, leads one to suspect that the 5-torus-orbifold will be generically anisotropic. This seems to be an important clue for the resolution of our puzzle of the previous section. To clarify it further, and to provide yet another consistency check on Eq.(3.4), we compare a suitable sector of the M-theory action compactified on $T^{5}$ and projected to $Z_{2}$ invariant states, with the relevant terms in the action of type IIB on K3. Start with the bosonic sector of the 11d supergravity (neglecting the Chern-Simons term, since after $Z_{2}$ projection this will only contribute coupling terms to scalars which we are ignoring):

$$
S_{M}^{(11)}=\int d^{11} x \sqrt{G^{(11)}}\left(R+\left|d A^{(3)}\right|^{2}\right)
$$

and choose the $11 \mathrm{~d}$ metric to be

$$
d s^{2}=G_{\mu \nu}^{(6)} d x^{\mu} d x^{\nu}+\sum_{i=7}^{11} e^{2 \gamma_{i}}\left(d x^{i}\right)^{2}+\ldots
$$

Here we have restricted ourselves to a rectangular 5-torus, so the angular terms do not appear. We also neglect the Maxwell field coming from the off-diagonal graviton, since this will be projected out by $Z_{2}$. The action in $6 \mathrm{~d}$ is then

$$
S_{M}^{(6)}=\int d^{6} x \sqrt{G^{(6)}} e^{\sum_{i} \gamma_{i}}\left(R+\sum_{i=7}^{11} e^{-2 \gamma_{i}}\left|d B^{i}\right|^{2}\right)+\ldots
$$


where $B_{\mu \nu}^{i}=A_{\mu \nu i}^{(3)}$ are the five 2-form fields in $6 \mathrm{~d}$.

To compare with type IIB on K3, recall that in this case there are altogether 21 antiself-dual and 5 self-dual $B$-fields, of which we set 16 of the anti-self-dual fields to zero since on the M-theory side they are expected to come from the twisted sector, over which we have no control. Combining the remaining 10 pairwise, we have 5 unconstrained $B$-fields left. The important point is that one of these comes from the NS-NS sector while the other 4 (as well as the 16 anti-self-dual ones that we neglected) are Ramond-Ramond.

There are also 25 scalars in the collection of multiplets that we are considering, but we set all of them to zero except the dilaton. Then on general grounds, the relevant part of the IIB action in $6 \mathrm{~d}$ will be

$$
S_{I I B}^{(6)}=\int d^{6} x \sqrt{g}\left[e^{-2 \phi}\left(R+\left|d B^{11}\right|^{2}\right)+\sum_{i=7}^{10}\left|d B^{i}\right|^{2}\right]+\ldots
$$

where $B^{11}$ has been chosen to be the unique NS-NS 2-form and the other $B^{i}$ are R-R.

If we now compare the two $6 \mathrm{~d}$ actions above, we can hope to express all the five compactification radii as functions of the dilaton, hence of the IIB string coupling. This relation will be valid only in the region of moduli space where all the other scalars have been set to zero, but it should serve as a qualitative guide to the relationship between the two theories. Moreover, whatever relationship emerges should of course satisfy Eq. (3.4) above.

Following a by now well-established procedure, we make a Weyl rescaling to relate the metric $G^{(6)}$ coming from M-theory and the metric $g$ of type IIB on K3:

$$
G_{\mu \nu}^{(6)}=e^{-\gamma} g_{\mu \nu}
$$

where $\gamma$ is to be determined. Matching the two sides leads to the set of equations

$$
\begin{aligned}
& -2 \gamma+\sum_{i} \gamma_{i}=-2 \phi \\
& \sum_{i} \gamma_{i}-2 \gamma_{11}=-2 \phi \\
& \sum_{i} \gamma_{i}-2 \gamma_{j}=0, \quad j=7, \ldots, 10
\end{aligned}
$$

whose solution is

$$
\begin{aligned}
\gamma_{11} & =\frac{2}{3} \phi \\
\gamma_{i} & =-\frac{1}{3} \phi, \quad i=7, \ldots, 10
\end{aligned}
$$


From the M-theory point of view the compactification radii are just $R_{i}=e^{\gamma_{i}}$. Thus we have found that

$$
\begin{aligned}
R_{11} & =\lambda_{6}^{2 / 3} \\
R_{i} & =\lambda_{6}^{-1 / 3}, \quad i=7, \ldots, 10
\end{aligned}
$$

with $\lambda_{6}=e^{\phi}$. It is reassuring that this set of values satisfies Eq.(3.4), as one can easily check.

At this point we see that (at least for the region of moduli space on which we are concentrating) the torus-orbifold scales anisotropically with $\lambda_{6}$. For weak coupling, four of the sides are very large and one is very small. Moreover,

$$
\frac{R_{11}}{R_{i}}=\lambda_{B}, \quad i=7, \ldots, 10
$$

so the ratio of the short side to the long ones is proportional to the string coupling. The conclusion that we draw from this is that for weak coupling, the torus is highly "squashed", and approximates a four-dimensional, rather than five-dimensional, manifold in this limit.

We can now propose, as a resolution to our puzzle of the previous section, that paradoxes based on equal distribution of Chan-Paton factors or their M-theory equivalents, among the 32 fixed points of $T^{5} / Z_{2}$ are not valid, since a regular 5-torus is highly nongeneric. Indeed, it is precisely in the region where the $6 \mathrm{~d}$ coupling is of order 1 (the "self-dual" point of the IIB string) where the torus regains isotropy, but this region is out of reach of perturbation theory (unlike both the strong and weak coupling regions, which can be exchanged by the S-duality contained in $O(5,21, \mathbb{Z}))$.

As the torus-orbifold degenerates, the 32 fixed points degenerate to 16 pairs with relative spacing of order the string coupling within each pair. So one may hope that M-theory on this space produces a multiple of 16 , and not necessarily 32 , copies of the twisted-sector multiplet. Thus it is consistent to assume that 16 chiral $N=4$ multiplets in $6 \mathrm{~d}$ are produced by M-theory in the twisted sector, as required to cancel gravitational anomalies.

The same phenomenon applies to the squashed $T^{5} / Z_{2}$ orientifold of the type IIA string to 5 dimensions. The computation of Ref. 25 for this case should lead to the conclusion that the $\left(U(1)^{16}\right.$ Chan-Paton group arises symmetrically since there are now only 16 and not 32 fixed points. (It may be worth studying this point more closely, though we will not do so here.) Thus the 5 d theory will have 16 extra $N=4$ Maxwell multiplets in 5 d, 
making a total of 21 (since 5 come from the untwisted sector). This is precisely what one gets from the type IIB theory on $K 3 \times S^{1}$ at generic points of the K3 moduli space.

If we turn on the other moduli, we can imagine more general degenerations of the squashed torus. For example, two or more sides could degenerate together. In these cases we will have $2^{5-k}$ fixed points where $k=2,3,4,5$ is the number of sides degenerating. Then of the 16 "missing multiplets", $2^{k-1}$ will come from each fixed point. From general principles of orientifolding, one expects that type IIA on such an orientifold should have enhanced gauge symmetries coming from Chan-Paton factors. Going back to M-theory, this provides an indirect argument that such squashed tori with $k \geq 2$ correspond to special points in the K3 moduli space, namely the points where IIA would acquire enhanced gauge symmetry or IIB would acquire a non-perturbative self-dual string of vanishing tension. Thus it is reasonable to believe that the "generic" $T^{5} / Z_{2}$ appropriate for compactification of M-theory to 6 dimensions is the one with a single squashed dimension.

It is noteworthy that anisotropic tori have recently played an important role 30 in understanding some apparent paradoxes in the proposed strong-weak coupling duality between heterotic and type I strings.

\section{Other Orbifolds of M-theory}

In 10 dimensions, orientifolds on $T^{n} / Z_{2}$ makes sense in type IIA for odd $n$ and type IIB for even $n$. M-theory is of course more closely related to type IIA than to type IIB. In particular, the $S^{1} / Z_{2}$ and $T^{5} / Z_{2}$ orbifolds of type IIA theory and M-theory have been discussed and compared in the preceding sections, and it is evident that there are both intriguing similarities and notable differences between the string and M-theory cases. Perhaps the most striking difference is that in the type IIA case one gets non-chiral theories (hardly surprising since one also lands in odd dimensions) but in M-theory the two known orbifolds lead to chiral string theories.

Here we investigate the remaining $T^{n} / Z_{2}$ orbifolds of M-theory, but will come to the disappointing conclusion that most of them break supersymmetry completely.

The first potentially interesting example is $T^{3} / Z_{2}$. In this case one might expect to find an interesting theory (although there will be no help from gravitational anomalies). However, the massless spectrum of bosonic fields in the untwisted sector is easily found to be $\left(g_{\mu \nu}, 3 B_{\mu \nu}, 7 \phi\right)$. This does not break up into supermultiplets, since $N=1$ supersymmetry in 8 dimensions admits only a single 2 -form field in the supergravity multiplet 
and none in the matter multiplet. Thus this orbifold breaks supersymmetry completely. This may appear surprising, since there must be a $T^{3} / Z_{2}$ orbifold of the type IIA string in 10d which preserves half the supersymmetries, namely the T-dual of the type I string on $T^{3}$ when all 3 directions are dualized. This theory would then be expected to agree with M-theory on $T^{3} / Z_{2} \times S^{1}$.

The way this paradox is avoided is that for the $T^{3} / Z_{2}$ orbifold of type IIA, the $Z_{2}$ action on the spacetime fields is

$$
\begin{aligned}
B & \rightarrow-B \\
A & \rightarrow-A \\
A^{(3)} & \rightarrow A^{(3)}
\end{aligned}
$$

(with $B, A$ and $A^{(3)}$ being the 2-form, 1-form and 3-form of the theory), unlike the orbifolds on $S^{1} / Z_{2}$ and $T^{5} / Z_{2}$ where the action is

$$
\begin{gathered}
B \rightarrow-B \\
A \rightarrow A \\
A^{(3)} \rightarrow-A^{(3)}
\end{gathered}
$$

The latter $Z_{2}$ action "lifts" to M-theory since $B$ and $A^{(3)}$ transform similarly, and they merge into a single 3 -form field in 11d. However, the former action does not, since $B$ and $A$ have different M-theoretic origins.

The $T^{7} / Z_{2}$ orbifold of M-theory similarly breaks supersymmetry completely. One heuristic way to think of this phenomenon is that for $T^{5} / Z_{2}$, the part of the transformation living in the first 10 dimensions is exactly the orbifold limit of K3 and has $S U(2)$ holonomy, while for $S^{1} / Z_{2}$ there is no such part and hence no holonomy. For the $T^{3} / Z_{2}$ and $T^{7} / Z_{2}$ cases, the part of the transformation visible below 10d corresponds to minus the identity matrix in one and three complex dimensions respectively, hence they do not have the right holonomies $(S U(1)$ and $S U(3)$ respectively) to preserve supersymmetry.

For $T^{9} / Z_{2}$ the situation is more interesting. From M-theory, one finds that the $Z_{2^{-}}$ invariant spectrum in $2 \mathrm{~d}$ contains 129 scalars. This may seem puzzling, but it comes from the fact that the metric in $2 \mathrm{~d}$, after removing its trace part, has formally -1 degree of freedom. So one of the 129 scalars should be combined with the metric, giving a supergravity sector with no on-shell degrees of freedom. Similarly, the 16 left-moving Majorana-Weyl gravitinos have formally -16 degrees of freedom, and combine with 16 right-moving Majorana-Weyl fermions to fit into the supergravity multiplet. The total 
gravitational anomaly [35] of this multiplet is proportional to $16 \cdot \frac{23}{24}+16 \cdot \frac{1}{24}$ which equals 16.

This leaves behind 128 scalars, which assemble into 16 real scalar multiplets of chiral $N=8$ supersymmetry: $\left(\phi^{i}, \lambda_{R}^{i}\right)$ with $i=1, \ldots, 8$. The anomaly of each of these multiplets is proportional to $8 \cdot \frac{1}{24}$ so altogether one has an anomaly of $\frac{16}{3}$. Combining the supergravity and matter multiplets, the total gravitational anomaly is proportional to $16+\frac{16}{3}=\frac{64}{3}$. This can be cancelled by 512 left-moving spin- $\frac{1}{2}$ fermions. Since there are now $2^{9}=512$ fixed points of the orbifold, it is conceivable that one left-moving fermion can come from each of the fixed points. These fermions would be singlets of the right-moving $(0,8)$ supersymmetryl

One piece of evidence to support this hypothesis comes from the expression for the coupling constant of the $2 \mathrm{~d}$ theory. Repeating the arguments given before but now for compactification down to an arbitrary number of dimensions $d$, one finds that the coupling constant in $d$ dimensions is given by

$$
\begin{aligned}
\lambda_{d} & =\frac{\lambda_{10}}{\sqrt{\operatorname{vol}\left(T^{10-d}\right)}} \\
& =\frac{\lambda_{10}^{1-(10-d) / 6}}{\sqrt{R_{d+1} \ldots R_{10}}} \\
& =\frac{R_{11}^{(d / 4)-1}}{\sqrt{R_{d+1} \ldots R_{10}}}
\end{aligned}
$$

For the special case $d=2$, one finds

$$
\lambda_{2}=\frac{1}{\sqrt{R_{3} \ldots R_{11}}}
$$

and remarkably, in this case the formula is totally symmetric in the 9 radii! This suggests that this is the unique situation where the torus is isotropic, and the twisted sector contribution can be expected to arise symmetrically from all the 512 fixed points. One may ask whether the resulting theory corresponds to a known compactification of the type IIB string down to 2 dimensions. The obvious choices $K 3 \times K 3$ and the Joyce 8-manifold of

1 An observation in an earlier version of our paper, that these fermions should lie in supersymmetry multiplets, has been corrected. 
$\operatorname{Spin}_{7}$ holonomy are ruled out since they preserve $1 / 4$ and $1 / 16$ of the supersymmetries respectively, while we want to preserve exactly half. Perhaps the right manifold is something like $\frac{K 3 \times K 3}{Z_{2}}$, but we will not pursue this point further herel.

The even-dimensional orbifolds of M-theory on $T^{n} / Z_{2}$ for $n=2,4,6,8$ do not correspond to orientifolds, but to the analogues of conventional orbifolds in string theory. The action is invariant under change of sign of an even number of directions without changing the sign of the 3 -form field. So we will not discover analogues of Chan-Paton factors in this case. Moreover, these orbifolds all land us in odd dimensions, where anomaly considerations are not useful.

In any case, for $n=2$ and $n=6$, supersymmetry is broken, while for $T^{4} / Z_{2}$ one finds that the untwisted sector decomposes into an $N=2$ supergravity multiplet and $3 N=2$ Maxwell multiplets. Comparing to the spectrum of M-theory on K3, we find precisely 16 missing Maxwell multiplets. So, if M-theory on $T^{4} / Z_{2}$ is to be the same as M-theory on $\mathrm{K} 3$ (in the appropriate orbifold limit of K3), then 16 Maxwell multiplets must come from the twisted sector. But these extra multiplets, if they appear, are not dictated by something as compelling as anomaly cancellation.

\section{Conclusions}

We have seen that one can define "orientifolds" of M-theory on $T^{n} / Z_{2}$ leading to supersymmetric theories for $n=1,5$ and 9 (the first of these cases was discovered in Ref.[14]). All lead to chiral theories and one can predict the twisted-sector states from considerations of cancellation of gravitational anomalies.

The $n=5$ case corresponds to type IIB on K3, though the compactification torus is generically anisotropic and virtually four-dimensional. This case should be worth studying in more detail than we have done here, particularly in order to understand the full correspondence between the moduli spaces for the M-theory and IIB compactifications. It is tantalizing that of the $S O(21,5)$ group occurring in the latter theory, the compactification torus for M-theory can only provide moduli for an $S O(5,5)$ part, while the twisted sector should provide another 16 degrees of freedom to enhance $S O(5,5)$ to $S O(21,5)$. This is reminiscent of Narain's observation [36] that for the heterotic string, the 16 dimensional internal-symmetry lattice in the heterotic string can mix nontrivially with the $(n, n)$

2 Actually, it is easy to check that the type IIB string on the orbifold $T^{8} / Z_{2}$ gives the right spectrum of states. 
spacetime lattice after compactification on an $n$-dimensional torus, to yield a $(16+n, n)$ lattice.

The interpretation of our results in terms of membranes of M-theory wrapping around the orbifolds, along the lines of Ref.[14], remains to be worked out.

Some of the interesting points raised by our analysis are the following. First of all, M-theory seems to easily yield chiral compactifications, reversing years of prejudice against 11-dimensional supergravity for its inability to provide chiral theories in lower dimensions. (This fact is also evident, although in a more subtle manner, from the fact that M-theory on a 2-torus gives the 10d type IIB string in a suitable limit 12]13]). So it should be worthwhile to investigate more sophisticated orbifolds than the ones we have considered (quotienting by groups larger than $Z_{2}$ ), particularly 7-dimensional ones, and see how this fits with the known Calabi-Yau compactifications of 10d string theory. Clearly this is much more difficult because of both the absence of gravitational anomalies in four dimensions and the reduced supersymmetry $(N=1)$.

The other intriguing question is what do we learn about the Chan-Paton factors, or rather their analogues, for M-theory. Evidently, whatever these are, they can produce $E_{8} \times$ $E_{8}$ gauge groups, and also 6-dimensional supermultiplets containing anti-self-dual 2-forms, both possibilities being out of reach of ordinary open strings. One line of thought might be to consider open membranes with free fermions living on their boundary, analogous to the interpretation of Chan-Paton factors for open strings in terms of free fermions living on the string boundary [37]. The worldvolume boundary field theory of the membrane would then be a two-dimensional conformal field theory of free fermions, and we know that with suitable spin structures this can indeed produce $E_{8}$ gauge groups, unlike the one-dimensional boundary theory of free fermions arising in open string theory. Whether this can be made more precise, and whether the same construction can yield anti-self-dual 2-forms in six dimensions, remains to be investigated.

\section{Note Added in Proof}

In a preprint that appeared on hep-th soon after this one, E. Witten[38] has independently made many of the observations in this paper, and also proposed an interesting picture of how the twisted sector states could arise from five-branes. After reading Ref. [38] we have made a small correction in Section 4 above, see the first footnote there.

\section{Acknowledgements}

We are grateful to Camillo Imbimbo and K.S. Narain for useful discussions. 


\section{References}

[1] E. Bergshoeff, E. Sezgin, and P.K. Townsend, Phys. Lett. B189 (1987) 75.

[2] M.J. Duff, P.S. Howe, T. Inami, and K.S. Stelle, Phys. Lett. B191 (1987) 70.

[3] P.K. Townsend, hep-th/9501068, Phys. Lett. B350 (1995) 184.

[4] E. Witten, hep-th/9503124, Nucl. Phys. B443 (1995) 85.

[5] I. Bars, hep-th/9503228, Phys.Rev. D52 (1995) 3567.

[6] J.A. Harvey and A. Strominger, The Heterotic String is a Soliton, hep-th/9504047.

[7] P.K. Townsend, hep-th/9504095, Phys. Lett. B354 (1995) 247.

[8] C. Hull and P. Townsend, hep-th/9410167, Nucl. Phys. B438 (1995) 109.

[9] M.J. Duff, J.T. Liu, and R. Minasian, Eleven Dimensional Origin of String/String Duality: a One Loop Test, hep-th/9506126.

[10] P.K. Townsend, P-Brane Democracy, hep-th/9507048.

[11] K. Becker, M. Becker, and A. Strominger, Fivebranes, Membranes, and Nonperturbative String Theory, hep-th/9507158.

[12] P. Aspinwall, Some Relationships Between Dualities in String Theory, hep-th/9508154.

[13] J.H. Schwarz, An SL(2,Z) Multiplet of Type II Superstrings, hep-th/9508143;

J.H. Schwarz, Superstring Dualities, hep-th/9509148;

J.H. Schwarz, The Power of M Theory, hep-th/9510086.

[14] P. Horava and E. Witten, Heterotic and Type I String Dynamics From Eleven Dimensions, hep-th/9510209.

[15] I. Bars and S. Yankielowicz, U-Duality Multiplets and Nonperturbative Superstring States, hep-th/9511098.

[16] M.J. Duff, H. Lu, C.N. Pope and E. Sezgin, Supermembranes With Fewer Supersymmetries, hep-th/9511162.

[17] J. Maharana, $M$ Theory and p-branes, hep-th/9511159.

[18] S. Kar, J. Maharana and S. Panda, Dualities in Five Dimensions and Charged String Solutions, hep-th/9511213.

[19] P.K. Townsend, D-branes from M-branes, hep-th/9512062.

[20] M.J. Duff, B.E.W. Nilsson and C. Pope, Phys. Lett B129 (1983) 39;

M.J. Duff and B.E.W. Nilsson, Phys. Lett B175 (1986) 417.

[21] M.J. Duff, in Architecture of Fundamental Interactions at Short Distances, Les Houches Lectures (1985), eds. P. Ramond and R. Stora (North Holland).

[22] P.S. Howe and P.K. Townsend, Supermembranes and the Modulus Space of D $=4$ Superstrings, in Supermembranes and Physics in $2+1$ Dimensions, eds. M.J. Duff, C.N. Pope and E. Sezgin (World Scientific 1990).

[23] A.C. Cadavid, A. Ceresole, R. D'Auria and S. Ferrara, hep-th/9506144, Phys.Lett. B357 (1995) 76 . 
[24] G. Papadopoulos and P.K. Townsend, hep-th/9506150, Phys.Lett. B357 (1995) 300.

[25] P. Horava, Nucl. Phys. B327 (1989) 461.

[26] A. Sagnotti, Open Strings and Their Symmetry Groups, in Non-perturbative Quantum Field Theory, Cargese 1987, eds. G. Mack et. al. (Pergamon Press 1988);

P. Horava, Phys. Lett. B231 (1989) 251;

J. Dai, R.G. Leigh and J. Polchinski, Mod. Phys. Lett. A4 (1989) 2073.

[27] P.K. Townsend, Phys. Lett. B139 (1984) 283.

[28] J. Schwarz, Gauge Groups for Type I Superstrings, in Lattice Gauge Theories, Supersymmetry and Grand Unification, Proc. Johns Hopkins Workshop, Florence 1982.

[29] J. Polchinski, hep-th/9407031, Phys. Rev. D50 (1994) 6041.

[30] J. Polchinski and E. Witten, Evidence for Heterotic-Type I String Duality, hepth/9510169.

[31] N. Seiberg, Nucl. Phys. B303 (1988) 286.

[32] P.S. Aspinwall and D.R. Morrison, String Theory on K3 Surfaces, hep-th/9404151.

[33] A. Dabholkar, hep-th/9506160, Phys.Lett. B357 (1995) 307.

[34] C.M. Hull, hep-th/9506194, Phys.Lett. B357 (1995) 545.

[35] L. Alvarez-Gaumé and E. Witten, Nucl. Phys. B234 (1983) 269.

[36] K.S. Narain, Phys. Lett. B169 (1986) 41.

[37] N. Marcus and A. Sagnotti, Phys. Lett. B188 (1987) 58.

[38] E. Witten, Five-branes and M-Theory on an Orbifold, hep-th/9512219. 